\title{
Blue eyes, brittle bones
}

\section{Adam Morton}

CASE

A woman aged 62 years had a telehealth appointment for the management of osteoporosis. An atraumatic crush fracture of the T8 vertebra had occurred recently, and three years earlier the patient had sustained a forearm fracture after falling from standing height. Her history was significant for mild episodic asthma requiring infrequent brief courses of oral glucocorticoids. Menarche occurred at age 15 years, and menses were regular until the age of 50 years. The patient drank 10-20 g of alcohol per day and had ceased smoking two years earlier. Her calcium intake was approximately $600 \mathrm{mg}$ per day. There was no family history of osteoporosis. Bone densitometry revealed T-scores of -3.4 and -3.1 in the lumbar spine and left femoral neck, respectively. Investigations had excluded secondary causes for osteoporosis, including primary hyperparathyroidism, hyperthyroidism, myeloma, coeliac disease, vitamin D deficiency and idiopathic hypercalciuria.

The woman's sclerae were noted to be discoloured during the telehealth consultation (Figure 1).

\section{QUESTION 1}

What is the most likely diagnosis?

\section{QUESTION 2}

What other features would be suggestive of this diagnosis?

\section{QUESTION 3}

How could a definitive diagnosis be made?

\section{QUESTION 4}

What are the treatment options?

\section{QUESTION 5}

What would be particularly important before commencing treatment of this patient?

\section{ANSWER 1}

The combination of osteoporosis and blue sclerae is suggestive of osteogenesis imperfecta type 1 . Currently 16 forms of osteogenesis imperfecta have been described with an estimated incidence of 1:10,000 births. ${ }^{1}$ Osteogenesis imperfecta type 1 is the mildest form and may present only as accelerated postmenopausal osteoporosis.

\section{ANSWER 2}

Osteogenesis imperfecta type 1 may be associated with dentinogenesis imperfecta (type 1), hearing loss (60\%) and excess or atypical fractures. ${ }^{2}$ More severe forms may be associated with short stature, bone deformity, scoliosis with resulting respiratory dysfunction, mitral and aortic valve regurgitation, joint and skin laxity, and easy bruising. ${ }^{1}$ Dentinogenesis imperfecta refers to discoloured and translucent teeth that wear rapidly and are prone to breakage and loss.

\section{ANSWER 3}

A definitive diagnosis can be made by demonstration of a mutation in the
COL1A1 or COL1A2 genes, present in $90 \%$ of individuals with osteogenesis imperfecta type $1 .^{3}$ Inheritance is autosomal dominant. Genetic screening enables a definitive diagnosis and family screening in the setting of incomplete penetrance of osteogenesis imperfecta with considerable variation in genotype-phenotype correlation and clinical manifestations, and the fact that no clinical feature is pathognomonic. Blue sclera have been described in 66 genetic syndromes and eight non-genetic syndromes, including iron deficiency anaemia, and in association with four medications. ${ }^{4}$ Type 2 and type 3 dentinogenesis imperfecta occur in the absence of osteogenesis imperfecta. Disadvantages of genetic screening include the cost of testing, that $10 \%$ of individuals with the disorder will not have a demonstrable mutation, possible implications for health insurance,

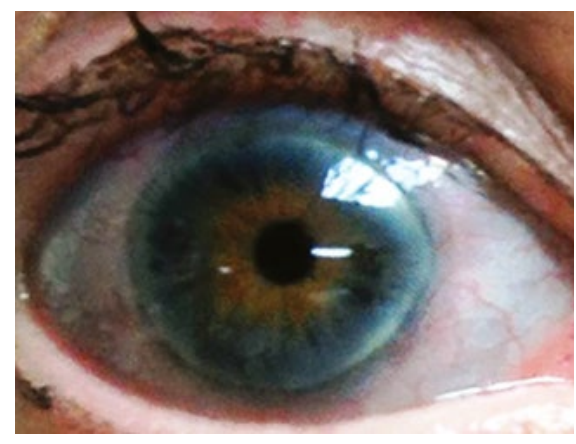

Figure 1. Scleral discolouration 
potential emotional burden for gene carriers who may never manifest features of the disorder, and the implications for pregnancy and affected offspring.

Other forms of osteogenesis imperfecta are associated with various genetic mutations and may be autosomal dominant or recessive. Prenatal testing in at-risk pregnancies may be performed by molecular genetic testing where a causative variant has been identified in an affected relative.

\section{ANSWER 4}

The management of osteogenesis imperfecta is multidisciplinary, including physiotherapy input for muscle strength, range of movement and mobility; pain management; regular monitoring of dentition and hearing; and management of bone disease. ${ }^{5}$ Bone quality is reduced as a result of defects in bone matrix and mineralisation. While there is strong evidence for the treatment of bone disease in children with osteogenesis imperfecta, the evidence for treatment of adults is considerably less, and management should be on a case-by-case basis, based on estimates of fracture risk. Other secondary causes of low bone density should be excluded, including vitamin D deficiency. Oral and intravenous bisphosphonates have been widely used in children and adults, demonstrating increased bone density and fracture reduction in children. Studies in adults were not powered to detect fracture reduction. Two small trials demonstrated improved bone density with teriparatide. No randomised trials have been performed regarding the efficacy of denosumab, and cases of hypocalcaemia and rebound hypercalcaemia have been reported following treatment, as well as rapid bone loss at the lumbar spine when the dose interval of denosumab was increased from three months to six months. ${ }^{6}$ A single phase 2 a trial of nine adults demonstrated that sclerostin inhibitors stimulated bone formation and increased bone density in people with osteogenesis imperfecta.

\section{ANSWER 5}

While to date no cases of bisphosphonaterelated osteonecrosis of the jaw have been reported in individuals with osteogenesis imperfecta requiring dental surgery, dental review is important prior to antiresorptive therapy. Notably, two cases of mandibular fracture have been reported as a result of dental extractions in children with osteogenesis imperfecta.

\section{CASE CONTINUED}

The patient gave a history of very poor dentition since her teens and was able to recall a professor of dentistry taking photos of her teeth and eyes. The patient did not have any difficulties with her hearing or easy bruising. Her nephew has similarly poor dentition. There was no family history of hearing loss, kyphoscoliosis or short stature. Secondary causes for osteoporosis were excluded. The result for genetic mutations testing was pending, and audiometry was planned for the future. The patient began annual infusions of zoledronic acid.

\section{Key points}

- Up to $30 \%$ of women with postmenopausal osteoporosis may have secondary factors contributing to bone loss. ${ }^{7}$

- Osteogenesis imperfecta type 1 should be considered in the differential diagnosis of postmenopausal osteoporosis.

- Blue sclera, poor dentition and hearing loss are all suggestive of a diagnosis of osteogenesis imperfecta.

- Bisphosphonates have the most evidence for the treatment of bone disease in osteogenesis imperfecta.

- Genetic testing for the disorder is available.

\author{
Author \\ Adam Morton FRACP, Endocrinologist and \\ Obstetric Physician, Mater Health and University \\ of Queensland, Qld \\ Competing interests: None. \\ Funding: None. \\ Provenance and peer review: Not commissioned, \\ externally peer reviewed. \\ Correspondence to: \\ Adam.Morton@mater.org.au
}

\section{References}

1. Lafage-Proust MH, Courtois I. The management of osteogenesis imperfecta in adults: State of the art. Joint Bone Spine 2019;86(5):589-93. doi: 10.1016/j.jbspin.2019.02.001.

2. Rauch F, Glorieux FH. Osteogenesis imperfecta. Lancet 2004;363(9418):1377-85. doi: 10.1016/ S0140-6736(04)16051-0.

3. Wenstrup RJ, Willing MC, Starman BJ, Byers PH. Distinct biochemical phenotypes predict clinical severity in nonlethal variants of osteogenesis imperfecta. Am J Hum Genet 1990;46(5):975-82.

4. Brooks JK. A review of syndromes associated with blue sclera, with inclusion of malformations of the head and neck. Oral Surg Oral Med Oral Pathol Oral Radiol 2018;126(3):252-63. doi: 10.1016/j. oooo.2018.05.012.

5. Ralston SH, Gaston MS. Management of osteogenesis imperfecta. Front Endocrinol (Lausanne) 2019;10:924. doi: 10.3389/ fendo.2019.00924.

6. Trejo P, Rauch F, Ward L. Hypercalcemia and hypercalciuria during denosumab treatment in children with osteogenesis imperfecta type VI. J Musculoskelet Neuronal Interact 2018;18(1):76-80.

7. Painter SE, Kleerekoper M, Camacho PM. Secondary osteoporosis: A review of the recent evidence. Endocr Pract 2006;12(4):436-45. doi: 10.4158/EP.12.4.436. 